\title{
Securely Deliver Data by Multi-path Routing Scheme in Wireless Mesh Networks*
}

\author{
Cao Trong Hieu and Choong Seon Hong ${ }^{* *}$ \\ Department of Computer Engineering, Kyung Hee University \\ Giheung, Yongin, Gyeonggi, 449-701 Korea \\ hieuct@networking.khu.ac.kr, cshong@khu.ac.kr
}

\begin{abstract}
Wireless Mesh Networks with static Transit Access Points (TAPs) have many advantages to connect different kinds of networks. While Mobile Ad hoc Networks still have many challenges to improve because of dynamic topology and security vulnerabilities, WMNs are the best solution for wireless communication recently. To utilize the characteristics of WMN's topology, in this paper, we propose an algorithm to preserve privacy for routing. This idea comes from the fact that if we can separate data traffic into more than one path, the probability to capture all traffic from intermediate node is very small. It means it is very difficult to launch traffic analysis attacks because of traffic confidentiality. In addition, to hide securely the real source and destination addresses a new technique is proposed along with an Adaptive Key Agreement Scheme. We apply Information Entropy to model our routing traffic and highlight the robustness of the algorithm. We also present a detail traffic evaluation observed from neighboring nodes to show the availability of our proposal in term of robustness, loop free and computational overhead.
\end{abstract}

Keywords: Security, Routing, Privacy Preservation, Information Entropy, Wireless Mesh Network.

\section{Introduction}

Along with Mobile Ad-hoc Network, Wireless Mesh Network recently has attracted increasing attention thank for the low-cost deployment and topology flexibility [2]. WMN represent a good solution to providing wireless Internet connectivity in a large scale. This new and promising paradigm allows for deploying network at much lower cost than with classic WiFi network. However, the routing mechanism must be secure.

We consider a Mesh Topology shown in Fig. 1. In this network, multiple mesh routers communicate with each other to form a multi-hop wireless backbone that forwards user traffic to the gateways which are connected to the Internet. Client devices access a stationary wireless mesh router at its residence.

Confidentiality (privacy) is one of the most important criteria regarding security aspect. In this paper, we focus on traffic confidentiality which prevents the traffic analysis attack from the mesh router.

* This work was supported by the MIC under ITRC Project (IITA-2006-C1090-0602-0002).

** Corresponding author. 
The key idea is if the traffic between source $S$ and destination D goes through only one route, any intermediate node can easily observe the entire traffic between S and D.

This route is vulnerable to traffic privacy attacks. To tackle this weakness, we propose a Multi-path routing mechanism which utilizes multiple paths for data delivery and can protect attacks based on data analysis.

The rest of the paper is organized as follows: section 2 briefly discusses some related works. In section 3, we propose an algorithm to find the multi-path between two mesh routers (nodes) when endusers want to communicate with each other or access to Internet. In addition, we propose an Adaptive Key Agreement Scheme to encrypt the data packets and transmit through multiple disjoint paths found in the previous step. In our scheme, we introduce a new technique that can hide real source and destination addresses. To make our

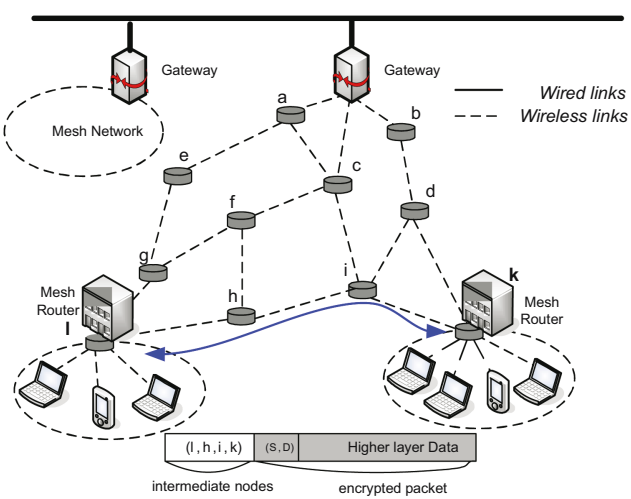

Fig. 1. General Mesh Topology proposal more reliable, we apply Information Entropy to model our routing traffic and prove the robustness of the algorithm in section 4. Finally, section 5 exposes some perspectives for further work.

\section{Related Work}

WMN is a hybrid network which has both mobile parts and stationary parts. However, due to limited capacity, delay constrains [3] and the lack of security guarantees [4, 10, 11], WMNs are not yet ready for wide-scale deployment. The first problem can be solved by using multi-radio and multi-channel Transit Access Points (TAPs) [5]. The other most important challenge concerned here is security especially in routing protocol.

In the existing literature, the onion routing [12] developed by David Goldschlag et al. can secure communication through an unpredictable path but it is necessary to encrypt message between routers. This means all intermediate nodes have to involve in encryption/decryption process which cause more overhead. In wireless ad-hoc networks, authors proposed schemes for location and identity privacy in [8, 9]. However, none of them can be applied to WMN directly. Traffic forwarding relationship among nodes is strongly dependent on their locations and the network topology, which is static in WMN. In addition, WMNs have some specifications that require adaptive changes in routing protocol. Our proposed routing protocol will take into account the inherent structure and solve those existing constrains.

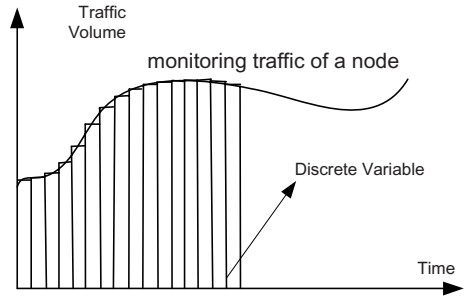

Fig. 2. Sampling continuous traffic 
In reality, the traffic of a node is a continuous function of time, as shown in Fig. 2.

However, in our proposal, to apply Information Entropy for privacy preservation, we consider the traffic as discrete random variable. Therefore, as the first step, we discrete the continuous traffic into piece-wise approximation of discrete values. Then we measure the amount of traffic in each period, usually in terms of number of packets, with assumption that the packet sizes are all equal.

\section{Proposed Algorithms}

In this part, we propose an algorithm to preserve privacy for routing along with Adaptive Key Management Scheme to transfer data securely between two nodes.

\subsection{Multi-path Finding Algorithm}

To apply our algorithm to routing protocol, it is needed a little bit change in routing table. We define Found Route to count and keep the number of paths found after the algorithm is executed. Node Occupied Status is 0 at initial stage and is set to 1 if a node is not available or it is already in a found route. Number_RREQ is the number of requests sent from source to destination. Each time a route is found or Request_Time is over, the source will send another request and Number_RREQ will be counted down. In our algorithm, Number_RREQ is equal to the number of neighbors of source node's AP. Request_Time can be assigned flexibly so that it is not too long to avoid overhead and not too short to guarantee path-finding process.

Hop count $(H C)$ is used to determine the shortest path and it is increased by 1 if $R R E Q$ or $R R E P$ is forwarded each hop. In this algorithm, $H C$ is also used to avoid $R R E Q$ 's loop back which also causes time and energy consumption.

In Step 1, all nodes' states are unoccupied. The RREQ is sent to all neighbors of source node. Node's availability [1] will be checked in this step. As mentioned above, Hop Count $(H C)$ is stored in routing table of each node and compared with new $H C$ index when a $R R E Q$ arrives. If new $R R E Q$ has $H C$ smaller than current one, the node will update new $H C$ and go to Step 2.

In Step 2, Node's Address is compared with Destination Address in RREQ. If it has the same address, Found Route is increased, Node Occupied Status is set to 1 and the number of $R R E Q$ is decreased by 1 . At this time, Number_RREQ and Request_Time are checked in Step 3 and if one of them equals 0, the algorithm is finished. Those conditions guarantee overhead avoidance.

Note that when a node does not satisfy the condition in Step 2, it will uni-cast back to notify the source and from this time it will not participate in the routing process. Moreover, the repetition of step 1 in step 2 is different from step 3 because the Number_RREQ is not counted down. Number_RREQ is only counted down when a new route is found. That is the reason why we need Request_Time to avoid overhead.

After the finding algorithm finished, in the routing table of involved nodes, the information about the number of routes and list of nodes in each route are stored. From that information, source node starts to send data through separate paths. As we discussed in [1], the path between source and destination in this case also need not be shortest path regarding hop count. 

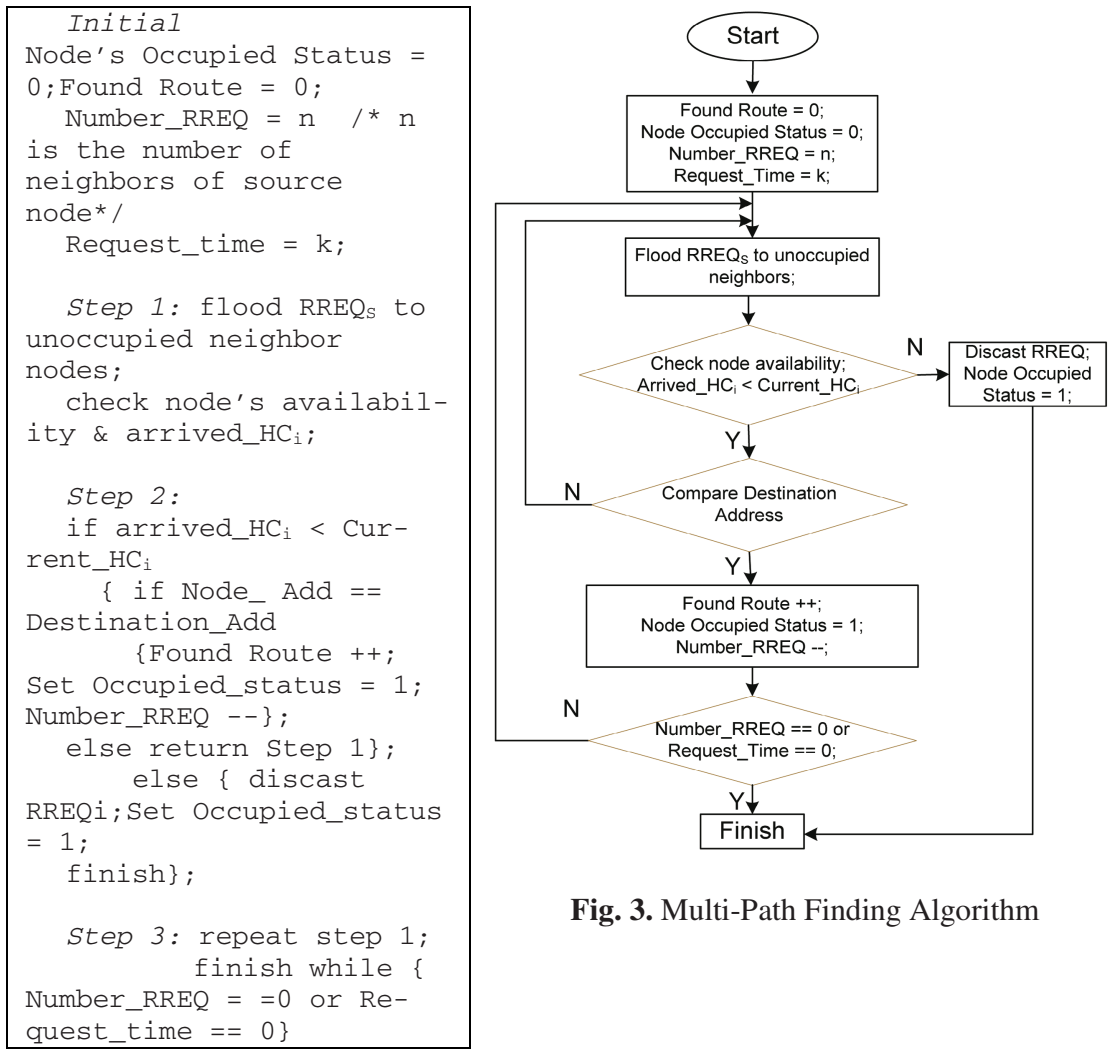

Fig. 3. Multi-Path Finding Algorithm

\subsection{Adaptive Key Management Scheme}

As briefly mentioned in section 1 , in this part, we introduce a new technique that can hide real source and destination addresses.

After process Multi-Path Finding Algorithm, the source and current source AP run 2party Diffie-Hellman in parallel with the destination and current destination AP do[13].

The key exchange includes 2 steps. At the first step (represented by solid arrows in Fig. 4), the source node and its access point (AP) choose a secret number $(x, y)$ respectively, a large co-prime $(g, p)$, and exchange to make a common key $K_{S / S^{\prime} A P}$ :

$$
K_{S / S^{\prime} A P}=g^{x y} \bmod p
$$

At this time, the destination node and its AP also choose a secret number $(u, v)$ respectively and exchange to make a common key $K_{D / D}{ }^{\prime} A$ :

$$
K_{D / D^{\prime} A P}=g^{u v} \bmod p
$$

In the second step (represented by dashed arrows in Fig. 4), the source AP and the destination AP run 2-party D-H in parallel with source and destination do and compute a common shared key $K_{S / D}$ :

$$
K_{S / D}=g^{x y u v} \bmod p
$$




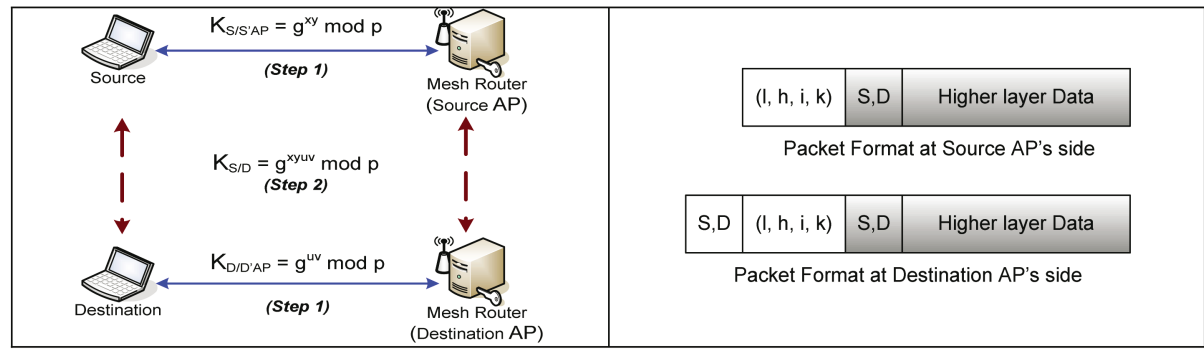

Fig. 4. Key Exchange Scenario

Fig. 5. Additional Field in Packet's Format

After this process, four nodes will have the same key $K_{S / D}$ and they can communicate securely.

To hide the real S/D addresses, we proposed a new technique that intermediate nodes can not extract to know address of S/D. This technique can prevent almost kinds of attacks based on data privacy.

At the source side, before transmitting, the data is split and encrypted with S/D addresses. After that, the addresses of intermediate nodes found in previous step (section 3) are attached without encryption. By this way, the intermediate nodes can only extract the source AP's address and destination AP's address. Each time an intermediate node receives a packet, it simply forwards this packet to the next hop in the address sequence. Without the need of knowing S/D address, all the packets will arrive to destination AP.

One challenge for proposed scheme is how to avoid computation overhead at receiver side because normally the destination AP will broadcast packets to all wireless clients in its range in MAC protocol. To solve this problem, the destination AP will use the common key $K_{S / D}$ to extract the S/D address in each packets and puts it in the unencrypted part before sending to its neighbor as showed in the figure 5 . When all clients receive the packets, they simply compare the destination address. If a packet is for a node, it can decrypt the packet thanks to $K_{S / D}$. If the packet is not for this node, it will drop and also can not try to decrypt the packet. In briefly, this technique can make the second protection layer for privacy of data not only at intermediate compromised nodes but also at receiver side. It also puts a little more computation overhead only at S/D access points.

To illustrate the privacy preserving and evaluate the rare probability that an attacker can capture and reassemble the data from source to destination in our algorithm, in the next section, we apply Information Entropy (also called Shannon Entropy) into our proposal.

\section{Traffic Evaluations}

In the information theory, the concept of Information Entropy (Shannon Entropy) describes how much information there is in a signal or event. In our proposal, it is used for evaluating the traffic volume that goes through separate routing paths described above.

We discrete continuous traffic into equal-size sampling period as discussed in the section 2 , and use $A$ as the random variable of this discrete value. The probability that 
the random variable $A$ is equal to $i$ (a node receives $i$ packets in a sampling period) is $P(A=i)$. Likewise, $P\left(B^{A}=j\right)$ is the probability that $B^{A}$ is equal to $j$. $(i, j \in N)$.

The Information Entropy of the discrete random variable $A$ is

$$
H(A)=\sum_{i=1}^{n} P(A=i) \log _{2}\left(\frac{1}{P(A=i)}\right)=-\sum_{i=1}^{n} P(A=i) \log _{2} P(A=i)
$$

$H(A)$ is a measurement of the uncertainty about the outcome of $A$. It means if the value of $A$ is distributed and no value predominates, $H(A)$ takes its maximum value. On the other hand, if the traffic pattern is Constant Bit Rate $(C B R)$, then $H(A)=0$.

Similarly, we have the entropy for $B^{A}$ as follows.

$$
H\left(B^{A}\right)=-\sum_{i=1}^{n} P\left(B^{A}=i\right) \log _{2} P\left(B^{A}=i\right)
$$

$B^{A}$ is a random variable representing the number of packets destined to node a observed at node $b$ in a sampling period.

Then we define the conditional entropy of random variable $B^{A}$ with respect to $A$ as

$$
H\left(A / B^{A}\right)=-\sum_{j=1}^{m} P\left(B^{A}=j\right) \sum_{i=1}^{n} p_{i j} \log _{2} p_{i j}
$$

in which, $p_{i j}=P\left(A=i \mid B^{A}=j\right)$ is the probability that $A=i$ given that $B^{A}=j$. $H\left(A \mid B^{A}\right)$ can be thought of as the uncertainty remained about $A$ after $B^{A}$ is known. The joint entropy of $A$ and $B^{A}$ can be shown as

$$
H\left(A, B^{A}\right)=H\left(B^{A}\right)+H\left(A / B^{A}\right)
$$

The mutual information of $A$ and $B^{A}$ which represents the information we can gain about $A$ from $B^{A}$ is defined as

$$
I\left(B^{A}, A\right)=H(A)+H\left(B^{A}\right)-H\left(A, B^{A}\right)=H(A)-H\left(A / B^{A}\right)
$$

Suppose the traffic observed at $b$ is proportional to $a$ at any sampling period. If $B^{A}$ $=j$, we can conclude that $A$ equals to a fixed value $i$. In this case, we have $P\left(A=i \mid B^{A}\right.$ $=j)=1$. This, according to $E q$. (III), makes the conditional entropy $H\left(A \mid B^{A}\right)=0$. It means the uncertainty about the outcome of $A$ when we know $B^{A}$ is 0 . From $E q$. $(V)$, we have $I\left(B^{A}, A\right)=H(A)$, implying that we gain the complete information about $A$, given $B^{A}$. Otherwise, if $B^{A}$ is independent of $A$, the conditional entropy $H\left(A \mid B^{A}\right)$ is maximized to $H(A)$. According to $E q$. $(V)$, we have $I\left(B^{A}, A\right)=0$, i.e., we gain no information $A$ from $B^{A}$. From $E q$. $(V)$, we also figure out that we have to minimize the maximum mutual information $I\left(B^{A}, A\right)$ that any node can obtain about $A$ to preserve privacy. In fact, since $B^{A}$ records the number of packets destined to node a, it can not be totally independent of random variable $A$. Therefore, the mutual information should be valued between the two extremes discussed above, i.e., $O<I\left(B^{A}, A\right)<H(A)$. This means that node $\mathrm{b}$ can still obtain partial information of $A$ 's traffic pattern.

Finally, we denote the average traffic through a node in a disjoint path as

$$
T_{\text {Avr }}=\frac{1}{m} \sum_{i=1}^{m} T_{i}
$$

in which, $m$ is the number of path found, $T_{i}$ is the traffic of a node at a specific time. 
We set up a simulation environment using $N S$-2 and analyze the traffic of an intermediate node by the data observed from its neighbor. We analyze traffic in three cases regarding the number of found route $(m=1,2,3)$. The traffic is randomly distributed through found route in previous step, and at the same time, the total traffic imultaneously runs through those paths is 100 percent. It means the more number of found routes, the less data is transferred through a node, and the probability to capture the whole traffic is very small.

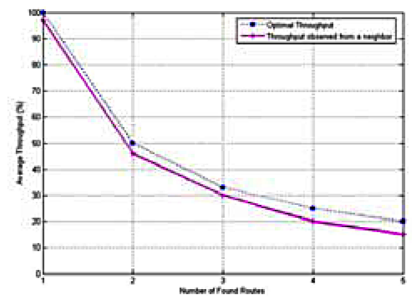

Fig. 6. Average Throughput Corresponding With Format Route

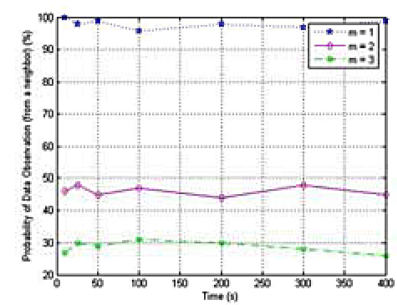

Fig. 7. Traffic observation Corresponding to Number of Found Routes

As shown in fig. 6, the obtained Average Throughput of a node in a route is always larger than the throughput observed by a neighbor of it.

In the figure 7, we monitor Traffic Throughput of a node by its neighbor in a period of time. The figure has shown that the probability of successfully capturing data will be reduced in direct proportion to the number of found routes $(m)$. It means traffic privacy will be preserved in direct proportion to $m$.

\section{Discussion and Conclusion}

Our proposed approach in this paper is applied to WMNs which have static Mesh Router. In case of Wireless Mobile Ad-hoc Networks, it is much more difficult to maintain found routes according to the node's mobility. In fact, the routers which placed in a building are supposed to be physically protected. Therefore, they are harder to attack than the Transit Access Points (TAPs) which are placed outside. Along with current key managements and authorization schemes, the APs are almost fully protected. If some attacks occur at intermediate nodes, as shown in previous sections, the probability that attackers can capture and restore data that is sent from source to destination through several disjoint paths is very small. Note that even if attackers can capture $99 \%$, they still can not merge the data and this stolen data is meaningless.

After a route was found, the data is split and marked before it is sent to the destination. When other routes are found, the remaining packets will be continuously sent through those paths randomly. This mechanism will reduce time consumption and also preserve data confidentiality.

In our algorithm, we especially concern about reducing overhead, so that we propose two parameters as Request_Time and Number_RREQ (discussed in section 3) to avoid time consumption. Also, the algorithm is loop free thanks to the discarded $R R E Q$ and the finish of participating progress of unavailable nodes in Step 2. 
The algorithm needs a small change in routing table and can be easily applied to the current routing platforms as discussed in section 2. Also, in our environment, there are enough number of nodes to find multiple disjoint path. Of course, in the worst case, there is only one communication path (for example with only 3 mesh routers) and this scenario becomes conventional communication (one route between source and destination).

In the future work, we will discuss attack scenarios and countermeasures regarding to security analysis and continue implementing our proposal in Testbed cooperating with existing routing protocol for WMNs. In addition, we will provide specific analysis how our scheme is implemented with well-known encryption algorithms to make the communication route more secure. Also, we are working on an algorithm for privacy preservation in Mobile Wireless PAN in which the network topology always changes due to node's mobility.

\section{References}

1. Cao Trong Hieu, Tran Thanh Dai, Choong Seon Hong.: Adaptive Algorithms to Enhance Routing and Security for Wireless PAN Mesh Networks, OTM Workshops 2006, LNCS 4277, pp. 585 - 594, 2006.

2. R. Karrer, A. Sabharwal, and E. Knightly.: Enabling large-scale wireless broadband: The case for taps, In HotNets, 2003.

3. V. Gambiroza, B. Sadeghi, and E. Knightly.: End-to-End Performance and Fairness in Multihop Wireless Backhaul Networks, Proc. MobiCom, 2004.

4. Ben Salem, N.; Hubaux, J.-P.: Securing wireless mesh networks, Wireless Communications, IEEE, April 2006 Page(s):50 - 55

5. M. Kodialam and T. Nandagopal.: Characterizing the Capacity Region in Multi-Radio Multi- Channel Wireless Mesh Networks, Proc. MobiCom, 2005.

6. M. G. Reed, P. F. Syverson, and D. Goldschlag.: Anonymous connections and onion routing, IEEE Journal on Selected Areas in Communications, 16(4):482-494, 1998.

7. Shu Jiang; Vaidya, N.H.; Wei Zhao.: Preventing traffic analysis in packet radio networks, DARPA Information Survivability Conference \& Exposition II, 2001. DISCEX '01, Proceedings Volume 2,12-14 June 2001 Page(s):153 - 158.

8. X. Wu and B. Bhargava.: Ao2p: Ad hoc on-demand position-based private routing protocol, IEEE Transactions on Mobile Computing, 4(4):335-348, 2005.

9. S. Capkun, J. Hubaux, and M. Jakobsson. : Secure and privacy preserving communication in hybrid ad hoc networks", Technical Report IC/2004/104, EPFL-DI-ICA, 2004.

10. Y.-C. Hu, A. Perrig, and D. B. Johnson.: Ariadne: A Secure On-Demand Routing Protocol for Ad Hoc Networks, In Proceedings of MobiCom, September 2002.

11. P. Papadimitratos and Z.J. Haas.: Secure Routing for Mobile Ad Hoc Networks, In Proceedings of CNDS, January 2002.

12. David Goldschlag, Michael Reed, Paul Syverson.: Onion Routing for Anonymous and Private Internet Connections, Communications of the ACM, Volume 42, Pages: $39-41$, February 1999

13. Asokan, N., and Ginzboorg, P.: Key agreement in ad-hoc networks, in Computer Communications, vol. 23, p. 1627 - 1637, 2000

14. Taojun Wu, Yuan Xue and Yi Cui.: Preserving Traffic Privacy in Wireless Mesh Networks, the International Symposium on a World of Wireless, Mobile and Multimedia Networks (WoWMoM'06), 2006, pp. 459 - 461. 